\title{
Re: Subramanian and Kumar. Vaccination rates and COVID-19 cases
}

\author{
Emilio Gianicolo ${ }^{1,2} \cdot$ Daniel Wollschläger $^{1} \cdot$ Maria Blettner $^{1}$
}

Received: 12 October 2021 / Accepted: 19 October 2021 / Published online: 24 December 2021

(C) The Author(s) 2021

We have read with interest the correspondence by Subramanian and Kumar [1] and found some major methodological issues which are worth discussing. The authors use an ecological approach to investigate the association of the percentage of population fully vaccinated with the trend in newly reported cases of positive SARS-Cov-2 tests between two consecutive 7-day time periods.

i. Basing the analysis entirely on data from two weeks instead of using the complete time-period since the beginning of the vaccination appears arbitrary.

ii. The analysis does not assess the size of the change in the number of positive test reports between the two time periods, but only evaluates whether a region had reported an increase in positive tests or not.

iii. Another issue arises in comparing countries with enormous differences in terms of testing capacities and/ or strategies, availability of vaccines, socioeconomic factors and demographic structures of the populations. One way to overcome this limitation could have been the stratification of the results according to continent awhile accounting for age. The lack of presentation of results makes their interpretation very challenging.

iv. Citing preliminary data from the CDC [2], the authors report an increase in the rates of hospitalizations and deaths amongst the fully vaccinated. However, this representation is incorrect as the $\mathrm{CDC}$ report rather evaluates the proportion of fully vaccinated among those hospitalized. The latter proportion is expected

Emilio Gianicolo

emilio.gianicolo@uni-mainz.de

1 Working Group for the Evaluation of Political Intervention, Division of Epidemiology and Health Services Research, Epidemiology and Informatics (IMBEI), Institute of Medical Biostatistics, University Medical Center of the Johannes Gutenberg-University Mainz, Obere Zahlbacher Str. 69, 55131 Mainz, Germany

2 Istitute of Clinical Physiology, National Research Council, Lecce, Italy to rise as the number of fully vaccinated people increases. Furthermore, this statistic is subject to Simpson's paradox as the vaccination rate among the elderly is particularly high, as is their risk for severe COVID-19 disease [3].

We agree that vaccination alone does not suffice as strategy to control the spread of the COVID-19 pandemic which instead requires integrating several measures [4]. However, since health outcomes among vaccinated and unvaccinated are not compared in a controlled individual-level study, increasing rates of deaths/hospitalizations even though vaccination rates improve does not, by itself, demonstrate a reduced efficacy of vaccines. In ecological studies, confounders and within-group misclassification may dilute, inflate, or even reverse any association. None of these limitations are mentioned or discussed.

In conclusion, although the authors correctly point out that vaccination alone may not be sufficient to reduce infection rates, their conclusions are not justified by their analysis. Further epidemiological evidence with individual information is needed to examine the efficacy of COVID-19 vaccination in real world studies.

Funding Open Access funding enabled and organized by Projekt DEAL.

Open Access This article is licensed under a Creative Commons Attribution 4.0 International License, which permits use, sharing, adaptation, distribution and reproduction in any medium or format, as long as you give appropriate credit to the original author(s) and the source, provide a link to the Creative Commons licence, and indicate if changes were made. The images or other third party material in this article are included in the article's Creative Commons licence, unless indicated otherwise in a credit line to the material. If material is not included in the article's Creative Commons licence and your intended use is not permitted by statutory regulation or exceeds the permitted use, you will need to obtain permission directly from the copyright holder. To view a copy of this licence, visit http://creativecommons.org/licenses/by/4.0/. 


\section{References}

1. Subramanian SV, Kumar A. Increases in COVID-19 are unrelated to levels of vaccination across 68 countries and 2947 counties in the United States. Eur J Epidemiol. 2021. https://doi.org/10.1007/ s10654-021-00808-7.

2. McMorrow M. Improving communications around vaccine breakthrough and vaccine effectiveness. 2021. https://fm.cnbc.com/ applications/cnbc.com/resources/editorialfiles/2021/07/30/CDC_ slides.pdf.

3. Morris J. Israeli data: How can efficacy vs. severe disease be strong when $60 \%$ of hospitalized are vaccinated? 2021. https:// www.covid-datascience.com/post/israeli-data-how-can-efficacyvs-severe-disease-be-strong-when-60-of-hospitalized-are-vacci nated.

4. Roberts S. The Swiss Cheese Model of Pandemic Defense. 2020. https://www.nytimes.com/2020/12/05/health/coronavirus-swisscheese-infection-mackay.html. Accessed 11.10.2021 2021.

Publisher's Note Springer Nature remains neutral with regard to jurisdictional claims in published maps and institutional affiliations. 